\title{
Évaluer la durabilité des exploitations agricoles. La méthode IDEA v4, un cadre conceptuel combinant dimensions et propriétés de la durabilité
}

Frédéric Zahm ${ }^{1, *}$, Adeline Alonso Ugaglia ${ }^{2}$, Jean-Marc Barbier ${ }^{3}$, Héloïse Boureau ${ }^{4}$, Bernard Del'homme ${ }^{5}$, Mohamed Gafsi ${ }^{6}$, Pierre Gasselin ${ }^{3}$, Sydney Girard ${ }^{1}$, Laurence Guichard ${ }^{7}$, Chantal Loyce ${ }^{7}$, Vincent Manneville ${ }^{8}$, Amandine Menet ${ }^{9}$ et Barbara Redlingshöfer ${ }^{10}$

${ }^{1}$ Irstea,UR ETBX, 50, avenue de Verdun, 33612 Gazinet Cestas, France

2 UMR Save, Bordeaux Sciences Agro,33170 Gradignan, France

3 Innovation, Univ Montpellier, CIRAD, INRA, Montpellier SupAgro, Montpellier, France

${ }^{4}$ Centre Ecodéveloppement de Villarceaux, La Bergerie, 95710 Chaussy, France

${ }^{5}$ Bordeaux Sciences Agro, 33170 Gradignan, France

${ }^{6}$ UMR LISST - Dynamiques Rurales, CNRS, UT2J, EHESS, ENSFA, Toulouse, France

7 UMR Agronomie, AgroParisTech, INRA, université Paris-Saclay, 78850 Thiverval-Grignon, France

${ }^{8}$ Institut de l'élevage (IDELE), 9, allée Pierre-de-Fermat, 63170 Aubière, France

9 CEZ - Bergerie nationale de Rambouillet, Parc du Château,CS 40609, 78120 Rambouillet, France

${ }^{10}$ INRA Agricultures urbaines / UMR SADAPT, Paris, France

\begin{abstract}
Résumé - Cet article présente le nouveau cadre conceptuel d'évaluation de la durabilité de l'exploitation agricole développé dans la méthode IDEAv4. Il combine une approche évaluative basée sur les objectifs assignés à une agriculture durable et une évaluation des propriétés des systèmes agricoles durables. Il s'ancre dans le champ de la durabilité forte, de la multifonctionnalité et prend en compte les enjeux globaux d'une agriculture durable. Ce cadre conceptuel a permis de construire 53 indicateurs permettant d'analyser la durabilité de l'exploitation agricole selon ces deux approches complémentaires. La première évalue la durabilité en organisant ces 53 indicateurs selon les 3 dimensions normatives du développement durable (agroécologique, socio-territoriale, économique), structurées en 13 composantes ; l'évaluation repose sur un système de notation basé sur 100 unités de durabilité pour chacune des 3 dimensions qui ne se compensent pas entre elles. La seconde évalue la durabilité en organisant les 53 indicateurs selon les 5 propriétés des systèmes agricoles durables (autonomie, robustesse, capacité productive et reproductive de biens et services, ancrage territorial et responsabilité globale) qui sont structurées de manière arborescente en 15 branches; l'agrégation des indicateurs y suit une démarche qualitative et hiérarchique mobilisant l'outil DEXi. Le potentiel pédagogique du concept de propriétés des systèmes favorise une approche transdisciplinaire de l'exploitation agricole. À la suite de ses trois précédentes versions, IDEAv4 renouvelle son potentiel d'usage pour accompagner la transition agroécologique.
\end{abstract}

Mots clés : IDEAv4 / évaluation de la durabilité / agriculture durable / propriétés de la durabilité / indicateur de durabilité d'une exploitation agricole

\begin{abstract}
Assessing the sustainability of farms. The IDEA v4 method, a conceptual framework based on the dimensions and properties of sustainability. This article presents the fourth version of the conceptual framework used in the "IDEA" method (Indicateurs de durabilité des exploitations agricoles or Farm Sustainability Indicators) (IDEAv4). It combines two approaches: one based on sustainable agriculture goals and the other applying a systemic approach focusing on the properties of sustainable agricultural systems. The IDEAv4 method includes both strong sustainability and agricultural multifunctionality paradigms. It takes into account the global challenges of sustainable agriculture. Using this framework, we were able to develop 53 indicators to assess the sustainability of a given farm
\end{abstract}

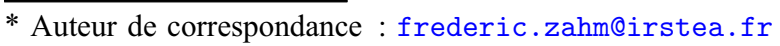


using two complementary approaches. The first approach assesses sustainability by dividing these 53 indicators into 13 components, according to the 3 normative dimensions of sustainable development (agroecological, socio-territorial, economic). This assessment process is based on a scoring system using 100 units for each dimension. There is no compensation between the scores of the three dimensions. The second approach assesses the level of sustainability based on 5 properties of sustainable agricultural systems (autonomy, robustness, ability to produce and reproduce goods and services, territorial embeddedness and overall responsibility), using a tree structure. This second approach is divided into 15 constituent branches of the 5 properties. The 53 indicators are aggregated with a qualitative and hierarchical approach, using the DEXi tool. This concept can be used for education, through a transdisciplinary teaching process. Following on from its three previous versions, IDEAv4 can also be used as a tool to support the process of agroecological transition.

Keywords: IDEA / Sustainability assessment tools / Sustainable agriculture / Properties of sustainability / Farm sustainability indicators

\section{Introduction}

La demande de méthodes d'évaluation de la durabilité des systèmes agricoles s'est accentuée ces dernières années pour permettre d'une part aux agriculteurs d'identifier les leviers d'action susceptibles d'améliorer la performance globale de leurs exploitations (niveau de durabilité), d'autre part au développement agricole d'élargir ses conseils techniques à l'aune de la durabilité et enfin à l'action publique d'évaluer ses dispositifs à l'aune de la transition agroécologique.

Les plus récents états de l'art (Schader et al., 2014 ; Lairez et al., 2015; De Olde et al., 2016) montrent une diversité importante des méthodes basées sur des indicateurs (une soixantaine recensées). Parmi celles-ci, la méthode IDEA (Indicateurs de durabilité des exploitations agricoles, Vilain et al., 2008 ; Zahm et al., 2008) est aujourd'hui l'une des quatre méthodes d'évaluation de la durabilité les plus utilisées dans l'Union européenne (De Olde et al., 2016). En effet, si son objectif initial était d'être un outil pédagogique au service de l'enseignement agricole et des agriculteurs pour rendre concret et mesurable le concept d'exploitation agricole durable, son usage s'est progressivement élargi à d'autres finalités telles que des travaux de recherche, l'accompagnement au changement et le conseil pour le développement agricole. Après l'avoir revisitée à deux reprises en 2003, puis en 2008, le comité scientifique de la méthode IDEA a conduit un travail de recherche renouvelant en profondeur son cadre conceptuel, ses grilles évaluatives et ses indicateurs, aboutissant à la méthode IDEA version 4 (IDEAv4). Cette refonte substantielle prend en compte :

- les propositions de modification issues de l'enquête nationale sur l'usage de la méthode IDEA (Rousselet, 2011);

- l'apparition de nouveaux enjeux sociétaux (alimentation, changement climatique, qualité de l'air, sobriété dans l'usage des ressources);

- l'évolution des cadres réglementaires publics et privés (normes, référentiels);

- les données les plus récentes de la statistique publique agricole pour définir les seuils de performance.

$\mathrm{Au}$ plan théorique, cette révision s'est appuyée sur une large revue de la littérature (Zahm et al., 2015) montrant le besoin d'une évolution du cadre conceptuel initial (1998) à l'aune de cadres analytiques complémentaires permettant une analyse élargie du concept de durabilité en agriculture. Cette révision s'est notamment traduite par l'intégration du concept de propriétés des systèmes durables, des principes de l'agroécologie et de l'économie circulaire, de l'action collective dans la transition écologique et d'une relecture des objectifs sociétaux alloués à l'agriculture (ONU, 2015).

Le cadre conceptuel d'IDEA v4 est basé sur la combinaison de deux approches évaluatives de la durabilité de l'exploitation agricole: l'une par les objectifs de l'agriculture durable et l'autre par les propriétés des systèmes agricoles durables. Cette combinaison aboutit au final à deux grilles de lecture évaluatives, structurées respectivement selon les 3 dimensions du développement durable (agroécologique, socio-territoriale et économique) et selon les 5 propriétés des systèmes agricoles durables (autonomie, robustesse, capacité productive et reproductive de biens et services, ancrage territorial et responsabilité globale). L'approche par les propriétés consolide la perspective systémique de l'exploitation agricole en introduisant une lecture transversale de sa durabilité.

La première partie de l'article resitue dans la littérature les deux approches mobilisées et présente le processus méthodologique mis en œuvre. La deuxième partie présente le nouveau cadre conceptuel d'IDEAv4 et ses deux grilles évaluatives mobilisant un noyau identique de 53 indicateurs. La troisième partie discute les apports, limites et nouveaux usages de la méthode avant de conclure sur ses perspectives de développement.

\section{Approches évaluatives de la durabilité de l'agriculture et démarche méthodologique}

Le cadre conceptuel d'IDEAv4 s'appuie sur la combinaison des deux approches évaluatives.

\subsection{Une approche évaluative basée sur les objectifs normatifs assignés à une agriculture durable}

Les cadres conceptuels basés sur des indicateurs centrés sur les objectifs d'une agriculture durable sont qualifiés de goal-oriented conceptual approaches (Von Wirén-Lehr, 2001) ou de goal-oriented frameworks (Alkan Olsson et al., 2009). Ils s'inscrivent dans une vision normative de la durabilité correspondant à «une capacité à réaliser un ensemble 
d'objectifs » (Hansen, 1996) et structurent une représentation de l'agriculture durable à partir des objectifs que celle-ci cherche à atteindre (Kates et al., 2005; Chia et al., 2009). Cette approche indique la direction à suivre pour aller vers une agriculture durable en fonction de la vision et des objectifs définis (Sala et al., 2015). Il s'agit de la dimension normative de la durabilité «qui discute ce sur quoi il y a accord et qui donne le cadre des priorités et actions à mettre en œuvre» (Hubert, 2004). Cette approche est pensée en fonction de valeurs que la société aspire à atteindre (Pope et al., 2004). Une telle représentation est en lien à la fois avec les défis sociétaux auxquels les agriculteurs et l'agriculture doivent faire face (durabilité étendue), mais aussi avec les objectifs internes à l'exploitation (durabilité restreinte). Cette approche est celle adoptée par l'ONU (2015) dans ses 17 objectifs de développement durable (ODD).

\subsection{Une approche évaluative basée sur les propriétés des systèmes agricoles durables}

Une seconde approche d'évaluation de la durabilité en agriculture s'appuie sur des cadres conceptuels mobilisant les propriétés de la durabilité (ou attributs ou principes). Les propriétés d'un système correspondent à des qualités émergentes non directement déductibles de ses sous-systèmes ou parties qui le composent. Elles sont issues de l'organisation du système de production, notamment des interactions entre ses sous-systèmes et des interactions avec son environnement (Gliessman, 2005). Ce sont les caractéristiques de ces interactions, plutôt que celles de ses parties, qui déterminent la durabilité du système analysé dans son ensemble. Qualifiée de system-based framework (Van Cauwenbergh et al., 2007) ou de systemic property oriented framework (Alkan Olsson et al., 2009), cette approche construit le choix argumenté d'indicateurs selon leur capacité à qualifier l'état d'un système au regard des propriétés systémiques de la durabilité (Conway, 1987; Bossel, 1999; Gliessman, 2005 ; López-Ridaura et al., 2005). Par exemple, une telle approche a été mobilisée par le programme de recherche MESMIS (Marco para la Evaluación de Sistemas de Manejo en Recursos Naturales incorporando Indicadores de Sustentabilidad) qui retient sept propriétés (productivité, stabilité, résistance, résilience, adaptabilité, équité, autonomie) pour qualifier la durabilité en agriculture sur des études de cas en Amérique latine (Astier et al., 2011).

\subsection{Démarche méthodologique}

\subsubsection{Processus de construction de la méthode}

Les 12 membres du comité scientifique IDEA représentent une diversité de disciplines: agronomie, économie, géographie, sciences de gestion, zootechnie. Ils ont conduit un processus de recherche pluriannuel structuré autour:

- d'une veille bibliographique permanente (scientifique et professionnelle) s'appuyant sur une analyse initiale détaillée de 60 méthodes d'évaluation de la durabilité ;

- de l'élaboration du cadre conceptuel;

- de la construction des grilles évaluatives et des indicateurs ;

- de l'apport d'avis extérieurs (chercheurs, enseignants, experts et utilisateurs).
Trois types de tests d'usage ont permis d'analyser les prototypes successifs, d'une part sur 130 exploitations agricoles représentant une large diversité de systèmes de production, de contextes territoriaux et de marchés, d'autre part lors de sessions de formation (étudiants, enseignants et professionnels) et enfin lors d'usages par des professionnels du conseil agricole.

Enfin, une double validation des seuils de performance des indicateurs a été mise en œuvre : d'abord par une confrontation systématique à la littérature, puis par un calcul complémentaire pour 14 indicateurs en utilisant trois bases de données nationales (du Réseau d'information comptable agricole, du Recensement agricole et de l'Agence de l'environnement et de la maîtrise de l'énergie).

\subsubsection{Explicitation des choix retenus}

Le choix et la construction des objectifs, propriétés et indicateurs ont fait l'objet d'un travail approfondi préalable, puis d'un débat et enfin d'un arbitrage. Ils reposent sur les deux principes suivants :

- être conforme au cadre théorique retenu;

- répondre à des standards de qualité (pertinence, parcimonie, fondement scientifique, robustesse, justesse d'analyse, mesurabilité, pédagogie, transparence) (Reed et al., 2006).

Dans leur construction, il a été adopté le principe que les indicateurs respectent les critères suivants: être facilement calculables par un agriculteur, un conseiller ou un étudiant, être transparents et dotés de seuils de performance adaptés à l'agriculture française pour guider l'interprétation. Le choix des 12 objectifs a été établi à partir d'une relecture des 18 objectifs d'IDEAv3 à l'aune de la littérature sur les nouveaux enjeux et objectifs du développement durable (ONU, 2015).

Une revue de littérature (12 articles) a permis d'identifier 36 caractéristiques (attributs) des systèmes durables que les auteurs ont regroupées en 5 propriétés en fonction de leur proximité conceptuelle: autonomie, robustesse, capacité productive et reproductive de biens et services, ancrage territorial et responsabilité globale.

Ce choix de ne retenir qu'un nombre limité d'objectifs et de propriétés résulte d'un compromis entre opérationnalité de la méthode et exigence de fidélité aux concepts retenus dans le cadre conceptuel. Le cadre d'analyse de chacune des 5 propriétés a fait l'objet d'une revue bibliographique conduisant à construire une carte heuristique (Fig. 1) structurée en branches et sous branches in fine déclinées en indicateurs. Le choix des indicateurs s'est appuyé sur la construction de la carte heuristique structurée selon les 5 propriétés de la durabilité conjuguée à la prise en compte des 12 objectifs. Il a débouché sur 53 indicateurs qui sont mobilisés dans leur ensemble et simultanément pour:

- l'évaluation des dimensions de la durabilité de l'exploitation agricole;

- l'évaluation des propriétés du système productif agricole.

Les méthodes de calcul des indicateurs résultent d'un compromis entre la capacité d'IDEA v4 à s'appliquer à tous les systèmes de production et la faisabilité de prendre en compte leurs spécificités. 


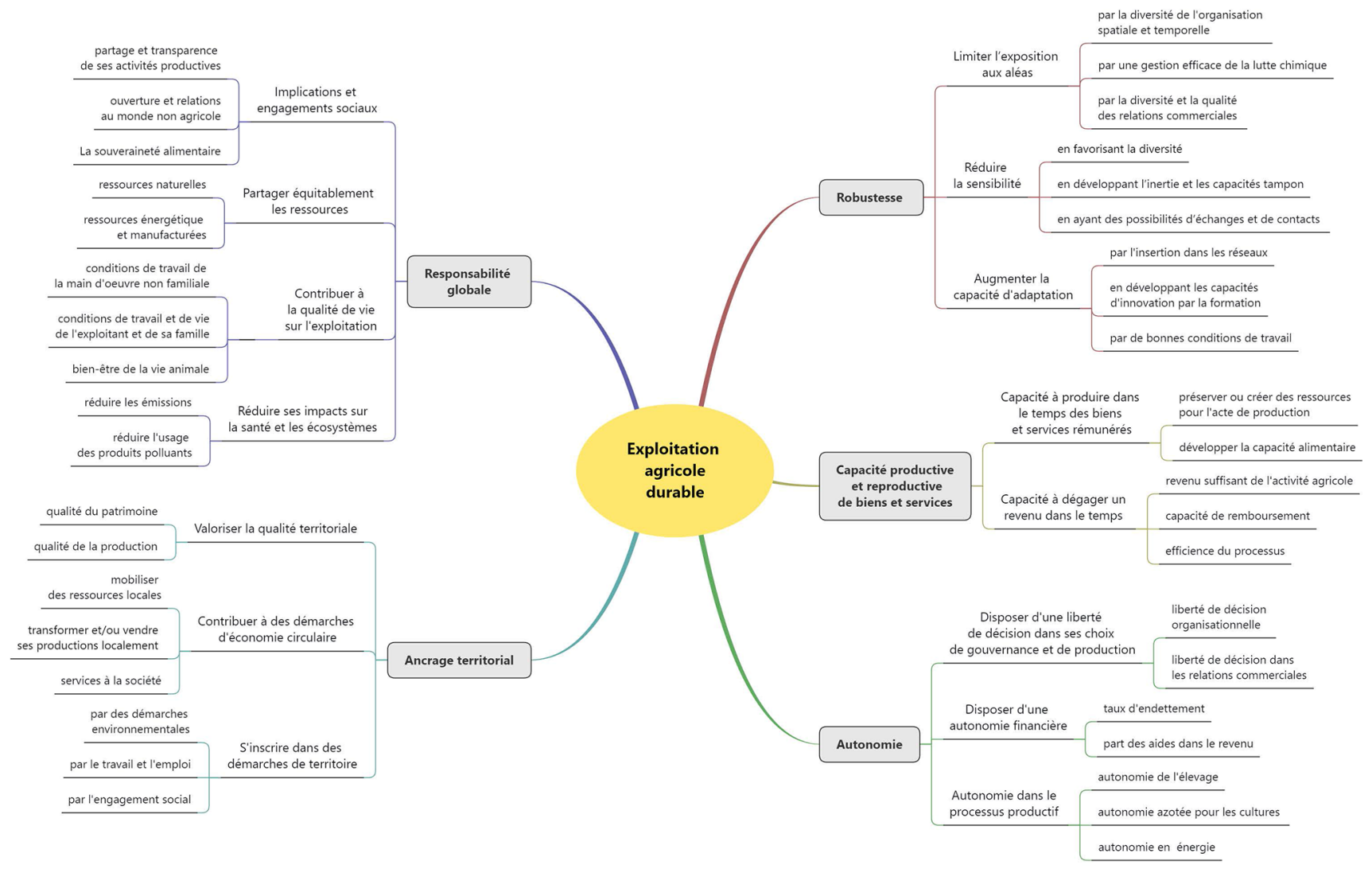

Fig. 1. Carte heuristique des 5 propriétés d'une exploitation agricole durable. Note: pour des raisons de lisibilité, cette carte heuristique n'est développée que jusqu'aux branches de niveaux 2 et ne présente pas les 53 indicateurs.

Fig. 1. Mind map of the 5 properties of a sustainable farm.

\section{Cadre conceptuel d'IDEA v4}

\subsection{Cadre théorique}

Notre revue de littérature montre que la structuration d'un cadre conceptuel d'évaluation de la durabilité basé sur une approche par les objectifs normatifs de la durabilité - cadre retenu dans la méthode IDEA v3 - reste nécessaire pour donner un sens concret aux objectifs à atteindre par une agriculture durable. Pour autant, si cette approche a montré tout son intérêt pédagogique et opérationnel, elle ne permet pas de caractériser, au plan systémique, l'état d'un système au regard de ses différentes propriétés (Binder et al., 2010). C'est pourquoi la structuration du cadre conceptuel d'IDEAv4 (Fig. 2) s'est enrichie d'une approche théorique par les propriétés des systèmes agricoles durables. Elle s'ancre dans le courant de la durabilité forte (Daly, 1990) qui rejette l'hypothèse d'une substituabilité ou compensation parfaite entre ressources naturelles et capital manufacturé. Pour qualifier ce concept de durabilité d'une exploitation agricole, 12 objectifs et 5 propriétés ont été retenus ( $c f . \S 2.3 .2)$.

Ces objectifs (Tab. 1) concernent à la fois la dimension agroécologique des activités agricoles mais aussi la dimension socioterritoriale de l'agriculture et la dimension économique de l'exploitation agricole. Ils renvoient à deux niveaux de durabilité tels que proposés par Terrier et al. (2013) :
Encadré 1. Définitions des concepts d'agriculture et d'exploitation agricole durables dans IDEAv4 (à partir de Zahm et al., 2015).

Une agriculture durable est une agriculture économiquement viable, écologiquement saine, socialement juste et humaine. Elle contribue, d'une part, à la durabilité du territoire dans laquelle elle s'ancre par la multifonctionnalité de ses activités et, d'autre part, à la fourniture de services environnementaux globaux (lutte contre le changement climatique, qualité de l'air, sécurité alimentaire, etc.).

Une exploitation agricole durable est une exploitation agricole viable, vivable, transmissible et reproductible inscrivant son développement dans une démarche sociétalement responsable. Cette démarche renvoie aux choix de l'agriculteur quant aux effets de ses activités et de ses modes de production au regard des objectifs propres à son exploitation mais aussi au regard d'objectifs externes à son exploitation renvoyant à des échelles socio-spatiales de niveau supérieur. Son développement repose sur 5 propriétés émergentes des systèmes agricoles durables: autonomie, robustesse, capacité productive et reproductive de biens et services, ancrage territorial et responsabilité globale. 


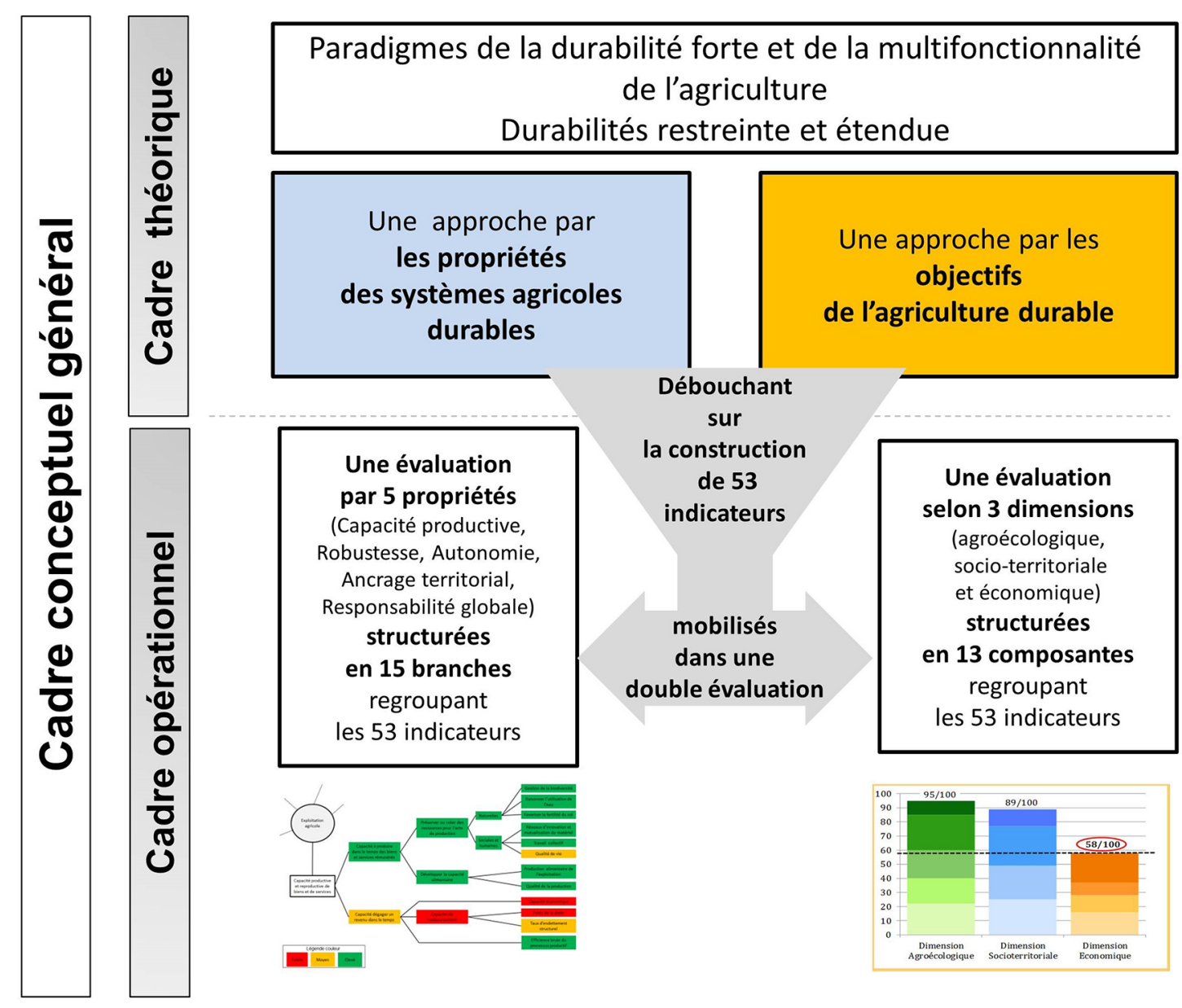

Fig. 2. Vue d'ensemble du cadre conceptuel de la méthode IDEAv4.

Fig. 2. Overview of the conceptual framework of the IDEAv4 method.

- la durabilité restreinte qui qualifie les objectifs autocentrés de l'agriculteur correspondant à ses facteurs internes de durabilité ;

- la durabilité étendue qui identifie les objectifs sociétaux d'une exploitation agricole contribuant au développement durable de niveaux d'échelles et d'organisations plus englobants (territoire, collectivité, pays, reste du monde) (Tab. 1).

Ils ont servi à construire le cadre paradigmatique en étant mobilisés lors de la définition des valeurs et visions partagées des auteurs ayant abouti à la définition des deux concepts : agriculture durable et exploitation agricole durable (Encadré 1).

Les 5 propriétés se définissent comme suit :

- l'autonomie d'une exploitation agricole correspond à sa capacité à produire des biens et des services à partir de ressources propres ou collectives locales (humaines, naturelles, physiques, cognitives, etc.), à permettre à l'exploitant agricole de disposer de sa liberté de décision et de développer des modes d'action permettant de limiter sa dépendance aux dispositifs de régulation publique (aides, quotas, droits à produire, ...) et aux acteurs de l'amont et de l'aval;

- la robustesse d'une exploitation agricole correspond à sa capacité à faire face à des variations (internes ou externes) de différentes intensités (fluctuations, perturbations, chocs) et de différentes natures (environnementales, sociales, économiques), et à conserver ou retrouver un état d'équilibre. Elle intègre de façon englobante les concepts de résilience, d'adaptation, de flexibilité ;

- la capacité productive et reproductive de biens et services d'une exploitation agricole correspond à sa capacité à produire et à reproduire dans le temps long, de manière efficiente, des biens et services, en dégageant suffisamment de revenu pour maintenir l'activité, sans dégrader sa base de ressources naturelles et sociales;

- l'ancrage territorial d'une exploitation correspond à sa capacité à contribuer à un processus de co-production et de valorisation de ressources territoriales. Il caractérise également la nature et l'intensité des liens marchands et non marchands que l'exploitation agricole construit avec son territoire, ses habitants, ses acteurs, son groupe social de vie;

- la responsabilité globale d'une exploitation correspond au degré d'engagement de l'exploitant agricole dans une démarche globale qui prend en compte les impacts environnementaux, sociaux et économiques dans ses choix de pratiques et d'activités. Cet engagement se structure autour de valeurs renvoyant à l'éthique et à l'équité. 
Tableau 1. Douze objectifs d'une exploitation agricole durable selon les niveaux de durabilité.

Table 1. Twelve objectives of a sustainable farm according to sustainability levels.

\begin{tabular}{ll}
\hline Niveaux de durabilité & Objectifs de l'agriculture durable \\
\hline Durabilité restreinte & $\begin{array}{l}\text { 1. Assurer la viabilité économique et la pérennité de l'exploitation } \\
\text { 2. Contribuer à la qualité de vie }\end{array}$ \\
$\begin{array}{l}\text { 3. Garder sa liberté d'action et son indépendance } \\
\text { 4. S'inscrire dans des démarches / engagements responsables éthiques }\end{array}$ \\
$\begin{array}{l}\text { 5. Produire et partager connaissances et savoir-faire } \\
\text { 6. Assurer le bien-être et la santé animale }\end{array}$ \\
$\begin{array}{l}\text { 7. Préserver les ressources naturelles (biodiversité, sol, eau, air) } \\
\text { 8. Préserver les ressources non renouvelables } \\
\text { 9. Préserver et/ou développer les paysages } \\
\text { 10. Répondre au défi du changement climatique (lutter contre et s'adapter) } \\
\text { 11. Contribuer à la sécurité et à la souveraineté alimentaire } \\
\text { 12. Contribuer à l'emploi et au développement territorial }\end{array}$ \\
\hline
\end{tabular}

\subsection{Cadre opérationnel}

Le cadre opérationnel (Fig. 2) décrit le processus d'agrégation du noyau de 53 indicateurs sélectionnés à partir de la construction de la carte heuristique. Ces mêmes indicateurs, agrégés selon les deux démarches évaluatives ci-après, apportent deux analyses complémentaires de la durabilité d'une exploitation agricole.

\subsubsection{L'approche évaluative selon les 3 dimensions de la durabilité}

Cette approche structure l'agrégation des indicateurs selon les 3 dimensions du développement durable (agroécologique, socio-territoriale, économique). Pour l'ensemble de ces 3 dimensions, l'agrégation des indicateurs se structure en 13 composantes qui ont les fonctions suivantes :

- organiser en thématique les 53 indicateurs ;

- donner un poids à chaque thématique de la dimension;

- permettre une compensation entre les notes des indicateurs d'une même composante.

La notation s'appuie sur un système d'unités de durabilité selon un barème adapté à chaque indicateur: les notes de chaque indicateur sont comprises entre la valeur zéro et une valeur plafond de durabilité maximale (Fig. 3). La note de chaque composante est la somme des notes de chacun de ses indicateurs, plafonnée à une valeur maximale comprise entre 20 et 35 selon les composantes. Ce plafonnement autorise une compensation entre les valeurs d'indicateurs d'une même composante. Il traduit le principe qu'il n'y a pas un modèle unique de durabilité mais des combinaisons sociotechniques et socioécologiques différentes: de nombreuses voies sont possibles pour atteindre un même niveau de durabilité. Ces règles de notation plafonnée permettent de prendre en compte la diversité des contextes (milieux humains et naturels), des systèmes de production et de leurs spécificités techniques. La note de chaque dimension est la somme de celle de ses composantes. La note maximale de chaque dimension est de 100 unités de durabilité correspondant au niveau le plus élevé de durabilité. L'absence de compensation entre composantes au sein d'une dimension implique d'obtenir la note maximale dans chaque composante pour atteindre le niveau le plus élevé de durabilité (100). La note finale de durabilité de l'exploitation correspond à la plus faible note des 3 dimensions (principe issu du courant de la durabilité forte impliquant la noncompensation entre les 3 dimensions) permettant également à l'agriculteur d'identifier où se situent ses marges ou ses besoins de progrès les plus élevés (exemple de la note de 58/100, Fig. 4).

\subsubsection{L'approche évaluative selon les 5 propriétés de la durabilité}

Dans cette approche, les mêmes 53 indicateurs sont organisés selon les 5 propriétés elles-mêmes constituées de 15 branches de «niveau 1 » (Figs. 1 et 5 ). Le premier principe adopté repose sur le choix de ne pas agréger les notes des 5 propriétés en une note globale de durabilité. En effet, une agrégation totale ne permettrait pas d'identifier des leviers d'action propres à chaque propriété et donc de discuter avec l'agriculteur des conditions d'une amélioration des propriétés les moins durables. Le second principe est une agrégation ascendante des indicateurs en passant par les différents nœuds intermédiaires des branches constitutives de chaque propriété (Fig. 5). Le troisième principe est une approche agrégative qualitative et hiérarchique mobilisant l'outil DEXi (logiciel d'aide multicritères à la décision) (Bohanec et al., 2008). Ce système de notation s'appuie sur l'attribution de classes de durabilité pour chaque indicateur: défavorable/intermédiaire/ favorable. L'agrégation au sein de chaque propriété est conduite pas à pas en suivant l'arborescence de la figure 1. Elle s'appuie sur des règles de décision structurées en tables. Elle permet de visualiser les résultats à l'échelle des branches intermédiaires comme montré à titre d'exemple à la figure 5 pour la propriété « Autonomie».

Pour conclure, ces deux démarches évaluatives sont complémentaires. L'approche évaluative par les 3 dimensions $\mathrm{du}$ développement durable (environnementale, sociale et économique) reste un référentiel méthodologique incontournable aussi bien pour la recherche que dans l'ingénierie du développement. Cependant, l'approche évaluative par les 5 propriétés permet de dépasser les impensés de la durabilité 


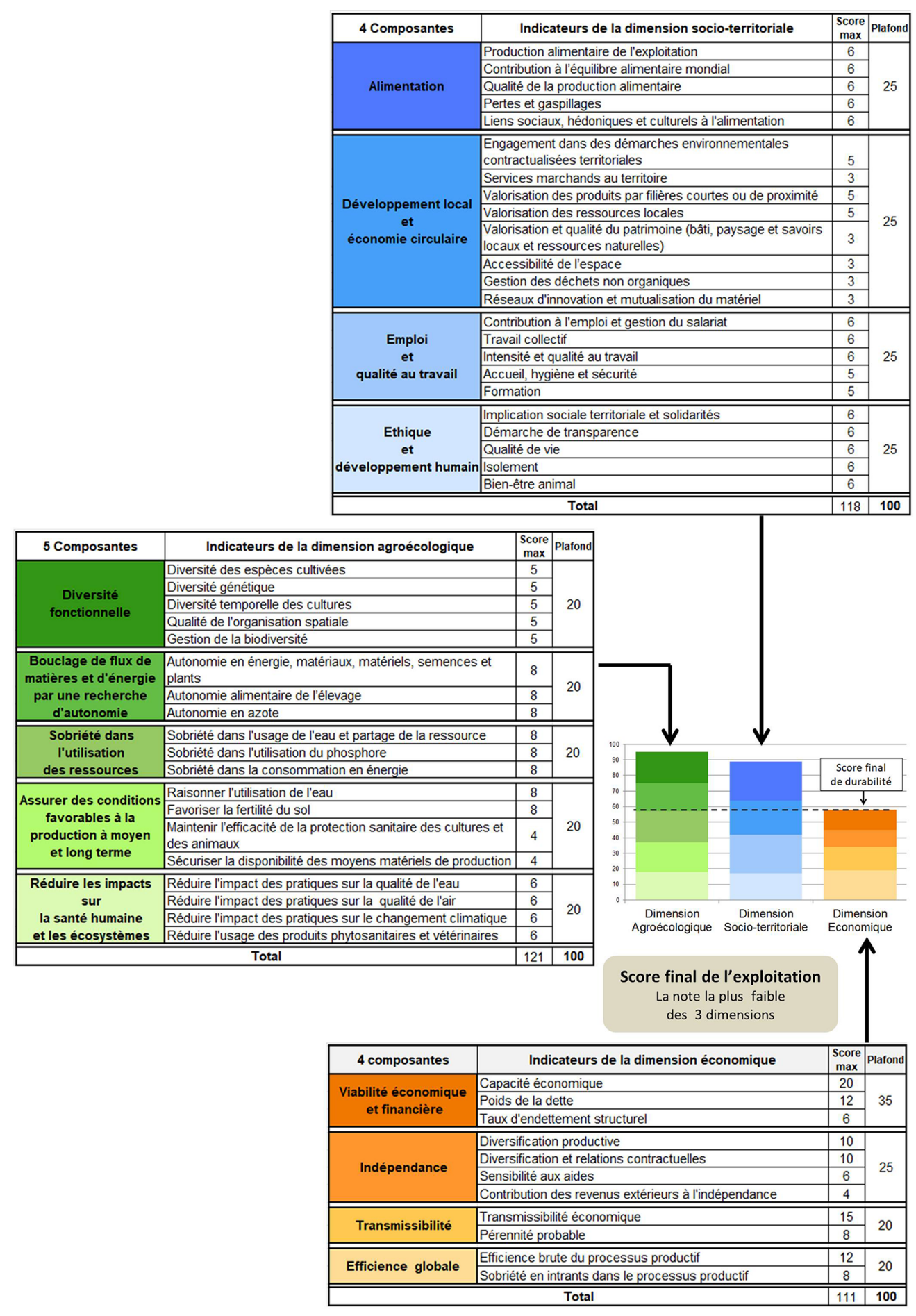

Fig. 3. Grille évaluative IDEAv4-approche par les 3 dimensions de la durabilité.

Fig. 3. IDEAv4 evaluation grid according to the 3 dimensions of sustainability.

telle que promue dans la vision normative du développement durable en 3 dimensions, à savoir mettre en exergue les questions d'autonomie, de robustesse, de capacité productive et reproductive de biens et services, d'ancrage territorial et de responsabilité globale. Elle consolide la conception systémique de l'exploitation agricole: la propriété est une émergence du système de production qui cristallise le fait que l'exploitation est un tout dont les performances sont plus que celles de ses parties. Les propriétés permettent une lecture transversale des dimensions de la durabilité (agroécologique, socio-territoriale, économique). Elles permettent d'interroger les synergies et les compromis entre chacune de ces dimensions. 

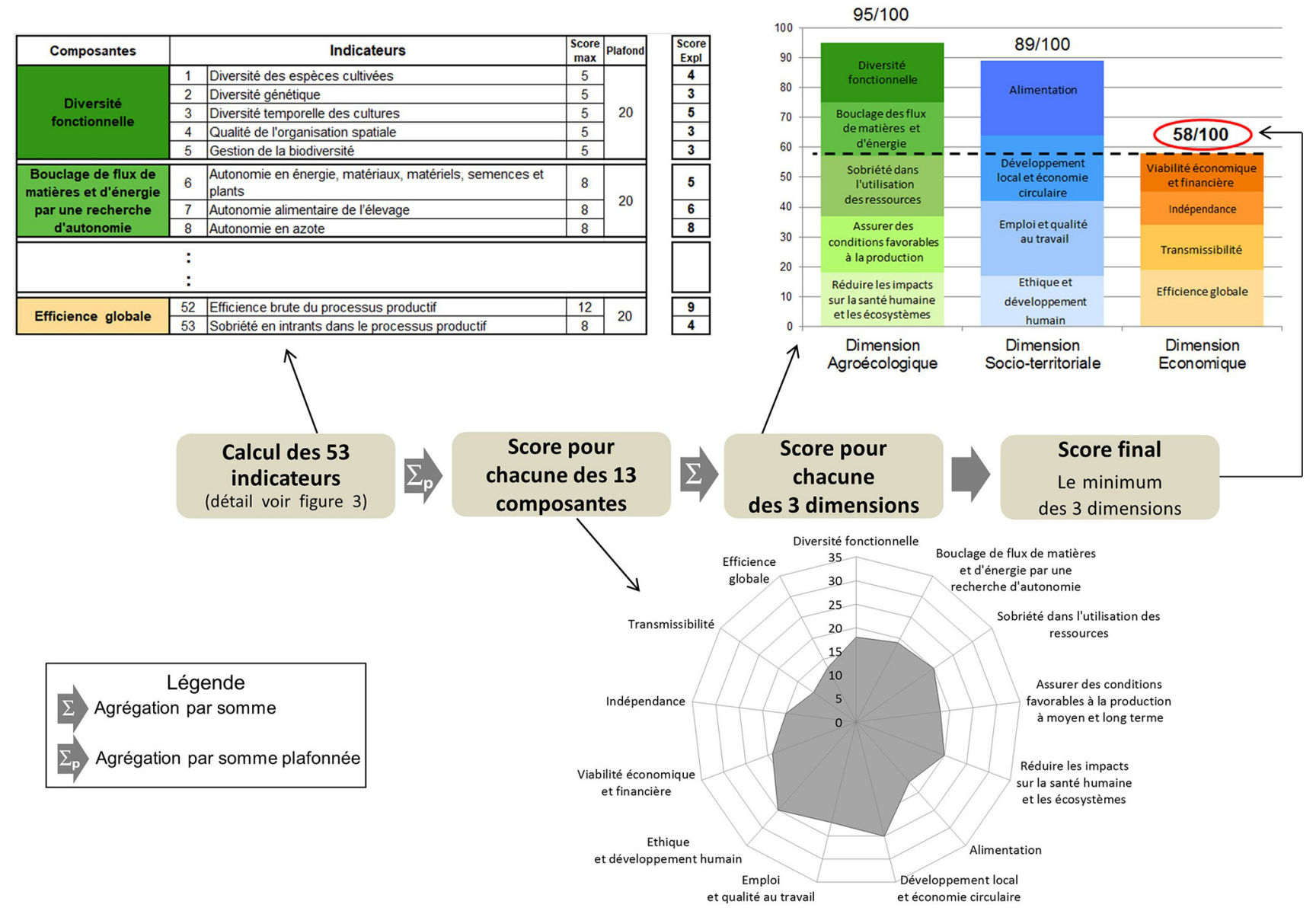

Fig. 4. Exemple de lecture évaluative du niveau final de durabilité d'une exploitation (selon les 3 dimensions de la durabilité).

Fig. 4. Example of evaluation of the final sustainability level for a farm (according to the 3 dimensions of sustainability).

\section{Discussion}

Les travaux sur le nouveau cadre conceptuel d'IDEAv4 ont été conduits avec les objectifs suivants :

- élargir son cadre théorique avec la prise en compte des propriétés des systèmes durables;

- maintenir sa finalité pédagogique pour un usage dans l'enseignement agricole;

- consolider son assise scientifique à partir des nouvelles connaissances disponibles depuis la parution d'IDEA 33 en 2008 ;

- conserver sa dimension opérationnelle reconnue dans le développement agricole (chambres d'agriculture, bureaux d'études, organismes nationaux à vocation agricole et rurale, etc.).

Ces travaux prennent en compte les nouveaux enjeux sociétaux (alimentation, changement climatique, qualité de l'air, sobriété dans l'usage des ressources) et enrichissent son cadre théorique pour analyser la durabilité (principes de l'agroécologie, économie circulaire, place de l'action collective, sobriété dans le processus productif).

Les précédentes versions d'IDEA ont été considérées comme «non participatives », dans le sens où les agriculteurs et les autres acteurs ne participent pas à la définition des objectifs de la durabilité (Binder et al., 2010). Dans IDEAv4, les principes essentiels de la méthode (propriétés, objectifs, durabilité forte, règles de calcul et pondération) ont été décidés par ses auteurs qui ont mis leurs propositions à l'épreuve de la critique et des enrichissements d'autres acteurs et utilisateurs au cours des six années de travail nécessaires à son développement. Par ailleurs, pour ses utilisateurs, la méthode conserve son principe de transparence basé sur l'accessibilité de l'ensemble des justifications (corpus théorique, guide méthodologique et référentiels) contrairement à d'autres méthodes livrées au format «boîte noire » ou réservées à un accès professionnel autorisé sous conditions. La méthode IDEAv3 est, avec la méthode Suisse RISE (Häni et al., 2003), la plus utilisée dans le monde du développement agricole et de la recherche en Europe (De Olde et al., 2016). La méthode IDEA v4, avec sa double évaluation par les dimensions et les propriétés, consolide la diversité potentielle de ses usages. Notre analyse de 60 méthodes évaluatives de la durabilité en agriculture confirme le caractère novateur d'une telle combinaison de deux grilles évaluatives (dimensions et propriétés) s'appuyant sur un même corpus d'indicateurs.

S'agissant de son usage pour l'enseignement, le nouveau cadre conceptuel refonde son potentiel pédagogique pour enseigner la durabilité de l'exploitation agricole en favorisant la transdisciplinarité (sciences agronomiques, sciences humaines et sociales) s'appuyant sur les caractéristiques systémiques et 


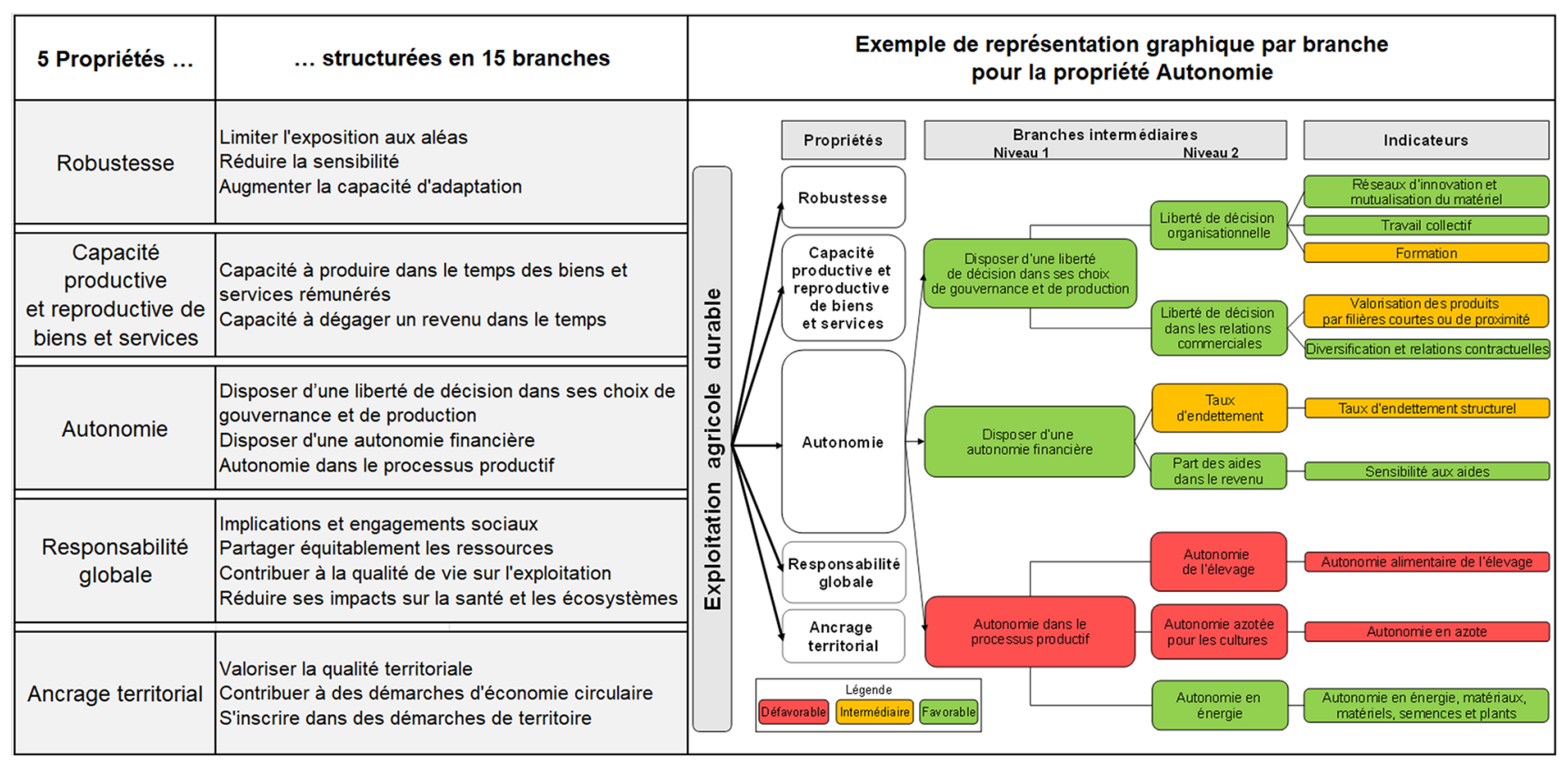

Fig. 5. Lecture évaluative des propriétés de la durabilité : exemple pour la propriété autonomie.

Fig. 5. Evaluative reading of sustainability properties: example for the property autonomy.

transversales de chaque propriété. Au plan pédagogique, l'évaluation qualitative par les propriétés est susceptible de lever également certaines réserves mises en avant sur le système de notation par les dimensions, qui pousserait ses utilisateurs à ne se référer qu'aux résultats chiffrés sans interpréter les notes à l'aune de la logique d'action de l'agriculteur.

S'agissant de son usage pour le conseil agricole, la méthode IDEA 44 peut aussi conduire à un renouvellement des pratiques, notamment pour accompagner la transition agroécologique, comme le montrent les premiers tests d'usage dans le conseil à des éleveurs laitiers s'engageant dans des actions de réduction d'émissions de gaz à effet de serre (Manneville et al., 2018). Pour l'analyse de ces changements, l'approche évaluative qualitative par les propriétés est en effet à même de favoriser une meilleure compréhension du fonctionnement systémique d'une exploitation par des conseillers, dont le métier et l'action restent à ce jour encore souvent cloisonnés par filière, ainsi qu'une analyse stratégique de l'exploitation (Capitaine et Jeanneaux, 2016).

La méthode IDEAv3 a été souvent utilisée, de manière partielle, dans l'action publique (certification Haute Valeur Environnementale, mesures agro-environnementales territorialisées, groupements d'intérêts économiques et environnementaux, marchés de service de qualité de l'eau, etc.). Les auteurs attirent l'attention sur le fait que ces usages impliquent systématiquement un test préalable et d'éventuelles adaptations. C'est pourquoi, les résultats fournis par les deux grilles évaluatives d'IDEAv4 sont à considérer comme un baromètre de la durabilité et non comme un mode opératoire d'un futur plan d'action ou comme un outil de contrôle administratif. Par ailleurs, il convient de souligner que la validité d'IDEAv4 s'inscrit, comme les précédentes versions, dans le contexte d'une agriculture française ou européenne. La méthode IDEAv3 a cependant été utilisée dans de nombreuses régions non européennes (Maghreb, Proche-Orient, Afrique de l'Ouest, Canada, Amérique latine, etc.). Si le cadre analytique d'IDEA v4 reste mobilisable quels que soient les types d'agriculture, les auteurs recommandent qu'une adaptation de la méthode soit étudiée au regard des spécificités locales (autres objectifs assignés à l'agriculture durable, conditions différentes au plan socio-économique ou de milieu, etc.).

Enfin, s'agissant de sa facilité d'usage, les tests réalisés pour l'approche par dimension montrent que la durée d'enquête pour la collecte des données n'excède pas quatre heures par exploitation. Le traitement et la mise en forme des données sont réalisés en une demi-journée contribuant à maintenir son opérationnalité. À la parution de l'article, des travaux restent à conduire pour que la méthode soit dotée de tout son outillage opérationnel : finaliser le calculateur de saisie de données et de restitution des résultats (pour la grille évaluative par dimensions), finaliser les règles d'agrégation des indicateurs de chaque propriété sous DEXi, développer son calculateur et le tester empiriquement (opérationnalité et pertinence pour l'action).

\section{Conclusion}

La formalisation d'un nouveau cadre conceptuel d'évaluation de la durabilité des exploitations agricoles porté dans IDEAv4 a donné lieu aux principaux apports suivants :

- une explicitation des propriétés d'un système de production agricole durable;

- l'affirmation d'un référentiel normatif basé sur 12 objectifs d'une agriculture durable pour qualifier les valeurs associées à ce concept ;

- une proposition d'évaluation de la durabilité de l'exploitation selon deux lectures, l'une par les 3 dimensions du développement durable (agroécologique, socio-territoriale et économique) et l'autre par les 5 propriétés de la 
durabilité (autonomie, robustesse, capacité productive et reproductive de biens et services, ancrage territorial et responsabilité globale).

Les perspectives de recherche sont orientées selon trois axes. Le premier vise à valider la capacité de cette nouvelle version à un enseignement transdisciplinaire de la durabilité auprès de l'enseignement agricole. Le deuxième questionne la capacité de cette double lecture évaluative à accompagner les acteurs du développement dans la voie vers la transition agroécologique, à en comprendre les effets et à en identifier les leviers d'action pour les exploitations agricoles. Le troisième analyse plus largement comment cette double approche évaluative contribue au management stratégique d'exploitations agricoles.

\section{Références}

Alkan Olsson J, Bockstaller C, Stapleton L, Ewert F, Knapen R, Therond O. 2009. A goal-oriented indicator framework to support integrated assessment of new policies for agri-environmental systems. Environ Sci Policy 12: 562-572.

Astier M, Speelman EN, López-Ridaura S, Masera OR, GonzalezEsquivel CE. 2011. Sustainability indicators, alternative strategies and trade-offs in peasant agroecosystems: analysing 15 case studies from Latin America. Int J Agricult Sustain 9(3): 409-422.

Binder CR, Feola G, Steinberger JK. 2010. Considering the normative, systemic and procedural dimensions in indicator-based sustainability assessments in agriculture. Environ Impact Assess Rev 30: 71-81.

Bohanec M, Messéan A, Scatasta S, Angevin F, Griffiths B, Krogh $\mathrm{PH}$, et al. 2008. A qualitative multi-attribute model for economic and ecological assessment of genetically modified crops. Ecol Modeling 215: 247-261.

Bossel H. 1999. Indicators for sustainable development: theory, method, applications, $118 \mathrm{p}$.

Capitaine M, Jeanneaux P. 2016. Agriculture en mouvement: innovations stratégiques et performance globale. Educagri Éditions, 120 p.

Chia E, Rey-Valette H, Lazard J, Clément O, Mathé S. 2009. Évaluer la durabilité des systèmes et des territoires aquacoles : proposition méthodologique. Cahiers Agricultures 18(2-3): 211-219.

Conway GR. 1987. The properties of agroecosystems. Agricult Syst 24: $95-117$.

Daly HE. 1990. Towards some operational principles of sustainable development. Ecol Econom 2: 1-6.

De Olde EM, Oudshoorn FW, Sørensen CA, Bokkers EA, de Boer IJ. 2016. Assessing sustainability at farm-level: lessons learned from a comparison of tools in practice. Ecol Indicators 66: 391-404.

Gliessman SR. 2005. Agroecology and agroecosystems. In: Pretty J (ed.), The Earthscan reader in sustainable agriculture. London: Earthscan, pp. 104-114.

Häni F, Braga F, Stämpfli A, Keller T, Fischer M, Porsche H. 2003. RISE - a tool for holistic sustainability assessment at the farm level, Int Food Agribusiness Manage Rev 6: 78-90.

Hansen JW. 1996. Is agricultural sustainability a useful concept? Agricult Syst 50: 117-143.
Hubert B. 2004. Agricultures et développement durable enjeux de connaissances et attitudes de recherche. Johannesbourg - L'INRA face au développement durable. Dossier Environnement INRA 27: 41-54.

Kates RW, Parris TM, Leiserowitz AA. 2005. What is sustainable development. Environ Sci Policy Sustain Develop 47(3): 8-21.

Lairez J, Feschet P, Aubin J, Bockstaller C, Bouvarel I. 2015. Évaluer la durabilité en agriculture. Guide pour l'analyse multicritère des productions animales et végétales. Quae, $231 \mathrm{p}$.

López-Ridaura S, Van Keulen H, Van Ittersum MK, Leffelaar PA. 2005. Multiscale methodological framework to derive criteria and indicators for sustainability evaluation of peasant natural resource management systems. Environ Develop Sustain 7: 51-69.

Manneville V, Girard S, Zahm F. 2018. Les propriétés de la durabilité en agriculture pour préparer la transition agroécologique face à l'enjeu du changement climatique, projet LIFE Carbon Dairy, IDELE, Collection Résultats.

ONU. 2015. Transformer notre monde: le Programme de développement durable à l'horizon 2030, New York.

Pope J, Annandale D, Morrison-Saunders A. 2004. Conceptualising sustainability assessment. Environ Impact Assess Rev 24(6): 595-616.

Reed MS, Fraser ED, Dougill AJ. 2006. An adaptive learning process for developing and applying sustainability indicators with local communities. Ecol Econom 59(4): 406-418.

Rousselet A. 2011. Durabilité des exploitations agricoles et méthode IDEA. Analyse de l'utilisation et perception de la méthode sur la période 2000 à 2010, mémoire d'ingénieur AgroSup Dijon sous la direction de F. Zahm (Irstea) et L. Guichard (INRA), 328 p.

Sala S, Ciuffo B, Peter Nijkamp P. 2015. A systemic framework for sustainability assessment. Ecol Econom 119: 314-325.

Schader C, Grenz J, Meier MS, Stolze M. 2014. Scope and precision of sustainability assessment approaches to food systems. Ecol Soc 19(3): 42.

Terrier M, Gasselin P, Le Blanc J. 2013. Assessing the sustainability of activity systems to support households farming projects. In: Marta-Costa A, ed. Methods and procedures for building sustainable farming systems: Application in the european context. Netherlands: Springer, pp. 47-61.

Van Cauwenbergh N, Biala K, Bielders C, Brouckaert V, Franchois L, Garcia V, et al. 2007. SAFE-A hierarchical framework for assessing the sustainability of agricultural systems. Agricult Ecosyst Environ 120(2-4): 229-242.

Vilain L, Boisset K, Girardin P, Mouchet C, Viaux P, Zahm F. 2008. La méthode IDEA Indicateurs de durabilité des exploitations agricoles, 3e édition, Dijon: Ed. Educagri, 184 p.

Von Wirén-Lehr S. 2001. Sustainability in agriculture - an evaluation of principal goal-oriented concepts to close the gap between theory and practice. Agriculture, Ecosyst Environ 84(2): 115-129.

Zahm F, Viaux P, Vilain L, Girardin P, Mouchet C. 2008. Farm sustainability assessment using the IDEA method. From the concept of farm sustainability to case studies on French farms. Sustainable Develop 16: 271-281.

Zahm F, Alonso Ugaglia A, Boureau H, Del'homme B, Barbier JM, Gasselin $\mathrm{P}$, et al. 2015. Agriculture et exploitation agricole durables : état de l'art et proposition de définitions revisitées à l'aune des valeurs, des propriétés et des frontières de la durabilité en agriculture. Innovations Agronomiques 46: 105-125.

Citation de l'article : Zahm F, Alonso Ugaglia A, Barbier J-M, Boureau H, Del'homme B, Gafsi M, Gasselin P, Girard S, Guichard L, Loyce C, Manneville V, Menet A, Redlingshöfer B. 2019. Évaluer la durabilité des exploitations agricoles. La méthode IDEAv4, un cadre conceptuel combinant dimensions et propriétés de la durabilité. Cah. Agric. 28: 5. 